\title{
BENEFÍCIOS DA INTELIGÊNCIA EMOCIONAL E SUAS IMPLICAÇ̃̃ES PARA A INTERVENÇÃO EM SAÚDE: EMPATIA E TRAÇOS DE PERSONALIDADE DOS ENFERMEIROS
}

\author{
Ana Galvão \\ Instituto Politécnico de Bragança; IUCISA: E \\ Bragança, Portugal \\ agalvao@ipb.pt \\ Maria José Gomes \\ Instituto Politécnico de Bragança; IUCISA: E \\ Bragança, Portugal \\ Fernando Pereira \\ Instituto Politécnico de Bragança; CIIE \\ Bragança, Portugal
}

Recepción Artículo: 19 de septiembre 2020

Admisión Evaluación: 2 octubre 2020

Informe Evaluador 1: 15 octubre 2020

Informe Evaluador 2: 17 octubre 2020

Aprobación Publicación: 27 noviembre 2020

\section{RESUMO}

Assistimos a vários debates sobre competências humanitárias em enfermagem, tais como: 0 cuidar, a compaixão e a empatia. Mantém-se em aberto a reflexão sobre se estas competências integram os traços da personalidade ou se podem ser ensinadas e treinadas. Há também o interesse por parte dos profissionais do ensino da enfermagem sobre como desenvolver estas competências nos estudantes. Assim, torna-se indispensável identificar qual o estado da expressão destas competências em profissionais de saúde. Realizamos um estudo exploratório, transversal, descritivo, correlacional e inferencial, com o objetivo de identificar os níveis de empatia e dos traços de personalidade dos enfermeiros; e, relações entre as variáveis socioprofissionais e as dimensões sob estudo, numa amostra de 232 enfermeiros: $186(80.2 \%)$ do género feminino; 149 (64.5\%) com mais de cinco anos de tempo de serviço. Utilizou-se um questionário online, constituído por questões socioprofissionais; pela Jefferson Scale of Physician Empathy; e o Big-Five Inventory (BFI-44). Constatamos que os enfermeiros apresentam níveis altos nas três dimensões da empatia: compaixão, perspetiva do doente e colocar-se no lugar do doente. Nos traços de personalidade as pontuações são acima da média para a extroversão, amabilidade, conscienciosidade e abertura; com exceção da dimensão neuroticismo onde a pontuação média é relativamente baixa. Encontraram-se correlações positivas, moderadas e estatisticamente significativas, entre as dimensões: tomada de perspetiva do doente; extroversão; amabilidade; conscienciosidade; e abertura. Verificaram-se diferenças estatisticamente significativas nas dimensões extroversão e compaixão, para a variável socioprofissional Género, obtendo-se pontuações mais elevadas nos homens, na extroversão e nas mulheres, na compaixão. Conclui-se 


\section{BENEFíCIOS DA INTELIGÊNCIA EMOCIONAL E SUAS IMPLICAÇÕES PARA A INTERVENÇÃO EM SAÚDE: EMPATIA E TRAÇOS DE PERSONALIDADE DOS ENFERMEIROS}

que os enfermeiros demonstram níveis altos nas dimensões da empatia. A empatia enquanto competência multidimensional, permite ao enfermeiro compreender sentimentos, necessidades e perspetivas dos doentes. No campo da saúde, é essencial para a prática profissional. Propomos a inclusão de um programa específico para a capacitação dos estudantes, pois irá ter impacto na satisfação e na qualidade dos cuidados prestados.

Palavras-chave: empatia; eersonalidade; enfermeiros

\section{ABSTRACT}

Benefits of emotional intelligence and its implications for health intervention: Empathy and personality traits of nurses. We have witnessed several debates on humanitarian skills in nursing, such as: caring, compassion and empathy. The reflection on whether these competences integrate personality traits or whether they can be taught and trained remains open. There is also an interest on the part of nursing teaching professionals on how to develop these skills in students. Thus, it is essential to identify the state of expression of these competencies in health professionals. We conducted an exploratory, cross-sectional, descriptive, correlational and inferential study, with the objective of identifying the nurses' levels of empathy and personality traits; and, relationships between socio-professional variables and the dimensions under study, in a sample of 232 nurses: 186 (80.2\%) were female; 149 (64.5\%) with more than five years of service. An online questionnaire was used, consisting of socio-professional issues; the Jefferson Scale of Physician Empathy; and the Big-Five Inventory (BFI-44). We found that nurses have high levels in the three dimensions of empathy: compassion, the patient's perspective and putting themselves in the patient's shoes. In the personality traits, the scores are above average for extroversion, kindness, conscientiousness and openness, with the exception of the neuroticism dimension where the average score is relatively low. Positive, moderate and statistically significant correlations were found between the dimensions: taking the patient's perspective; extroversion; kindness; conscientiousness; and openness. There were statistically significant differences in the dimensions of extraversion and compassion, for the socio-professional variable Gender, with higher scores in men, in extraversion and in women, in compassion. It is concluded that nurses demonstrate high levels in the dimensions of empathy. Empathy as a multidimensional competence allows nurses to understand patients' feelings, needs and perspectives. In the health field, it is essential for professional practice.

Keywords: empathy; personality; nurses

\section{INTRODUÇÃO}

"Não sei o que nos espera, mas sei o que me preocupa: é que a medicina, empolgada pela ciência, seduzida pela tecnologia e atordoada pela burocracia, apague a sua face humana e ignore a individualidade única de cada pessoa que sofre, pois embora se inventem cada vez mais modos de tratar, não se descobriu ainda a forma de aliviar o sofrimento sem empatia ou compaixão." João Lobo Antunes

Atualmente assistimos com frequência a várias reflexões sobre competências de relação terapêutica em enfermagem, tais como: a Humanitude, a comunicação, a relação de ajuda, a compaixão e a empatia. Continuam a existir dúvidas sobre se essas competências são inatas ou se podem ser ensinadas/aprendidas. Há também 0 interesse por parte dos profissionais do ensino da enfermagem sobre como desenvolver estas competências nos estudantes. Assim, torna-se indispensável identificar qual o estado atual destas competências.

Para contextualizar o construto de empatia, focámo-nos na Teoria do Cuidado Humano de Margaret Jean Watson, a qual, tem aprofundado a sua teoria continuadamente. Em 1979, teve lugar a primeira publicação da sua teoria (Watson, 1979). De forma continuada têm sido desenvolvidos alguns aspetos, tendo sempre como meta a valorização de dimensões para além do campo concreto, com ênfase nas perceções subjetivas e experiências do outro.

Passamos a descrever os conceitos que advoga Watson, e como estes conceitos se articulam com a competência de empatia. A teórica considera que 0 cuidado será efetivo, através do relacionamento transpessoal, ou 
seja, o cuidado que transcende tempo, espaço e matéria do paciente e do enfermeiro para construírem um único elemento em sintonia, além do momento pontual da interação, de maneira a favorecer a restauração (healing). Frequentemente os cuidados de enfermagem privilegiam, a dimensão física, com a execução de procedimentos técnicos cada vez mais modernos. Mas na dimensão transpessoal, o cuidado de enfermagem é capaz de aceder aos aspetos emocionais e subjetivos do doente, de forma a objetivar a transpessoalidade, através da comunicação em saúde, da empatia e da compaixão. Estas ferramentas podem desenvolver e manter a relação terapêutica, a harmonia e a confiança necessárias para o processo do cuidar.

A Teoria do Cuidado Humano de Watson integra dez elementos direcionados à relação do Cuidar. Estes elementos, na sua maioria, estão relacionados de forma direta com a empatia e com a Humanitude. Por exemplo, o elemento: estar presente nas relações, espelha o fundamento da empatia e da Humanitude. Segundo a teoria, é a partir do foco e da atenção dispensada ao outro que o processo empático se inicia e que torna possível a compreensão da experiência do doente. Estamos presentes onde está a nossa atenção. Se ao cuidarmos estivermos preocupados com outras questões e ou situações não relacionadas com o outro que está à nossa frente, dificilmente conseguiremos desenvolver e demonstrar empatia, sublinha a autora.

Os elementos oito e nove estão relacionados com a promoção do equilíbrio do ambiente e dos doentes, respetivamente. A postura empática, que acolhe e aceita o outro, é capaz de influenciar fortemente estes aspetos. 0 comportamento empático tanto pode provocar uma mudança num ambiente desfavorável, como pode atender às necessidades físicas, mentais e emocionais dos doentes. Reconhecer o que num ambiente é hostil para o outro, colocar-se no seu lugar e optar não só por intervenções técnico-procedimentais, mas por uma postura que 0 modifique, está no cerne da restauração e atendimento das necessidades previstas nesses elementos.

Sobre 0 elemento dez, recai a reflexão de como a atitude empática auxilia nos aspetos relacionados à espiritualidade, à vida e à morte. Como colocar-se no lugar do outro que está num processo de finitude.

Segundo Watson, um dos instrumentos mais adequados para estabelecer e manter a relação de ajuda e confiança entre enfermeiro e doente é a empatia. Advoga ainda que, a partir do genuíno interesse no processo do cuidar, é possível desenvolver uma relação empática, quando se reconhece o doente como quem vivencia uma experiência única e 0 enfermeiro expressa compreensão e aceitação através de linguagem verbal e não verbal. As evidências mostram que, a Teoria do Cuidado Humano de Watson, quando implementada, é capaz de contribuir para a satisfação e autonomia do doente, favorecendo também a dignidade humana através do cuidado ético e humano.

Encontramos nas evidências que o desenvolvimento da empatia é influenciado por diversos fatores, destacando-se os fatores internos (fatores genéticos, aspetos do desenvolvimento neural e variáveis de temperamento), e os fatores externos ou de socialização, como imitação, estilos parentais e estilo de relacionamento pais e filhos, conforme ilustrado na Figura 1. 
Figura 1 Fatores de influência e consequências do desenvolvimento da empatia (Mcdonald \& Messinger, 2011)

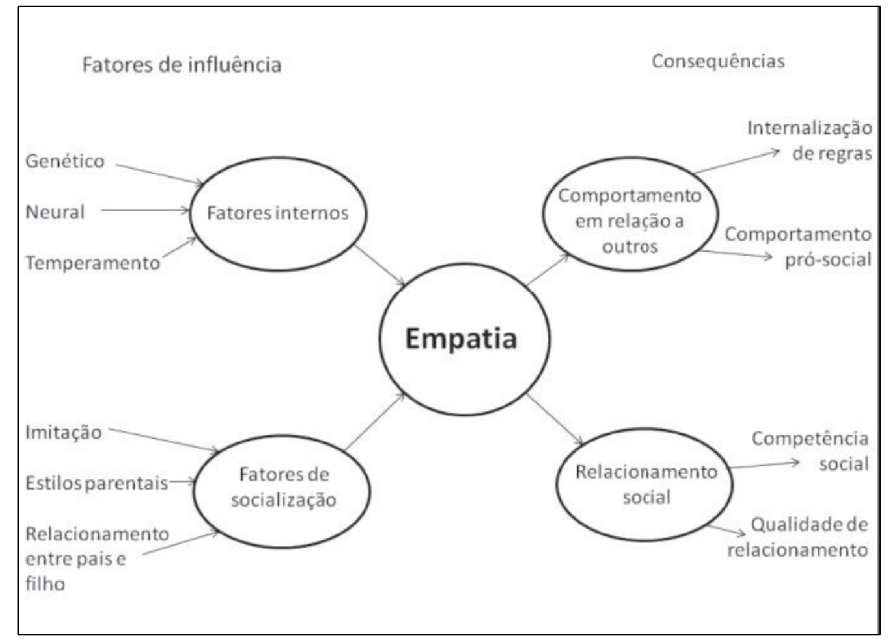

Não há um consenso teórico sobre a definição operacional de empatia. Adotámos neste trabalho a definição elaborada por Hojat et al. (2002), visto que abrange os principais pontos comumente enfatizados em estudos sobre esta temática.o autor define, empatia como um atributo cognitivo que envolve a habilidade de entender e suportar as experiências e perspetivas internas do paciente e a capacidade de comunicar esse entendimento. Há autores que fazem uma distinção entre as diferentes formas que 0 indivíduo utiliza para poder compreender 0 outro a partir da perspetiva deste. Mencionando que a empatia compreende dois componentes: emocional e cognitivo. 0 componente emocional seria a capacidade de experienciar algo semelhante ao sentimento do outro, estando relacionada com a discriminação perceptiva. 0 enfermeiro, observa os sinais emocionais do doente, como a expressão facial, postura corporal e tom de voz, e de forma condicionada sente a mesma emoção. Considera-se também que a perceção dos sinais emocionais no outro pode lembrar ao sujeito experiências passadas, quando ele já experienciou esta emoção e esta memória fá-lo sentir como quem ele observa. 0 componente cognitivo diz respeito à dimensão intelectual dos estados internos e pensamentos do outro, ou seja, a capacidade de compreender a situação a partir da perspetiva do outro. Para tal, é necessário que o enfermeiro/estudante de enfermagem, desenvolva a consciência de que existe um outro e que este possui pensamentos e sentimentos diferentes dos seus.

Assim, sentir empatia envolve compreender as reações emocionais e sentimentos do doente, ou seja é imperativa a capacidade de colocar-se no lugar do doente ou sentir empatia através duma experiência emocional semelhante já vivenciada. Contudo isto não define de forma linear que o enfermeiro evidencie empatia, pois há outras variáveis inerentes, como por exemplo os seus traços de personalidade. Nas fontes consultadas, identificam-se duas diferentes e possíveis reações empáticas: uma, e a tendência em agir pro-socialmente, quando 0 indivíduo utiliza esta capacidade de compreensão para ajudar o doente a aliviar o desconforto e o sofrimento. A outra e o sofrimento pessoal no qual, ao observar o desconforto do doente, o enfermeiro tende utilizar estratégias para aliviar o próprio sofrimento. A primeira reação, também denominada de consideração pelo outro, e o pressuposto teórico mais utilizado para explicar as respostas de empatia ao considerar que esta e um processo interno e o comportamento pro-social de ajuda e uma possível consequência externa com mensuração possível.

0 conceito de empatia possui várias vertentes, no entanto, todas consideram a capacidade de compreender os sentimentos de outra pessoa e o comunicar de tal experiência, sempre baseada nos pilares cognitivos, afetivos e comportamentais. Para 0 desenvolvimento do comportamento empático, destaca-se 0 real interesse com a 
preocupação do sofrimento alheio, representando um processo consciente, que visa 0 aperfeiçoamento das relações interpessoais através da consolidação do vínculo afetivo e das habilidades comunicacionais. Peplau (1997) deu o seu valioso contributo para a qualidade do Cuidar, e definiu enfermagem como um processo significativo, terapêutico e interpessoal que visa promover a saúde das pessoas e comunidades através do desenvolvimento da personalidade com vista a alcançar progressos a nível criativo, construtivo, produtivo, pessoal e comunitário. Peplau, concebe esta relação, como sendo interpessoal, desenvolvendo-se entre uma pessoa doente ou que necessita de cuidados de saúde e um enfermeiro com formação especializada para reconhecer e responder ao pedido de ajuda. Diz-nos (Chalifour, 2008)que o processo de doença acontece, quando o confronto com as necessidades, as frustrações, os conflitos e a ansiedade não permitem à pessoa reorganizar e reconstituir eficazmente a sua estrutura de personalidade. Numa relação terapêutica, para 0 enfermeiro conseguir ajudar o doente, é fundamental que consiga mobilizar não só os saberes e as competências adquiridos, mas também os pressupostos que constituem os principais recursos dessa relação nomeadamente: a empatia, a congruência e a consideração positiva incondicional.

No Regulamento do Perfil de Competências do Enfermeiro de Cuidados Gerais (Ordem dos Enfermeiros, 2011), podemos ler que é competência do enfermeiro estabelecer relações terapêuticas com o cliente e/ou cuidadores, através da utilização de comunicação apropriada e capacidades interpessoais.

Sempre que refletimos sobre a relação de ajuda, utilizamos a perspetiva rogeriana, a qual define empatia como um processo, complexo, exigente e intenso, ainda que subtil e suave, através do qual o enfermeiro, imaginariamente, se coloca no papel e na situação do doente, de modo a interpretar os seus sentimentos, atitudes e comportamentos.

Para Goleman (1995) a empatia emerge da autoconsciência, ou seja, quanto maior for o conhecimento que o enfermeiro tem das suas emoções, maior será a sua capacidade de intuir os sentimentos do doente. Aqui observa-se uma sintonia com o terceiro elemento da Teoria do Cuidado Humano de Watson, o qual diz respeito ao cultivo de práticas que melhorem 0 autoconhecimento. A manutenção de atividades que desenvolvam 0 autoconhecimento é incentivada pelos teóricos que analisam a empatia, pois ele é fundamental para o movimento interno que proporciona a capacidade de se colocar no lugar do outro. Sendo uma das competências do enfermeiro no domínio do desenvolvimento das aprendizagens profissionais, desenvolver 0 autoconhecimento e a assertividade.

Nesta perspetiva teórica de Goleman, a empatia, enquanto habilidade do enfermeiro para reconhecer o que sente e vivencia o doente, reside na observação e interpretação da sua comunicação não-verbal. Sustentando-se em estudos fisiológicos, o autor concluiu que só existe empatia quando a comunicação não-verbal do doente e do enfermeiros está sincronizada e, sugere, que para o enfermeiro ser empático é necessário estar calmo e recetivo para que os sinais, mais subtis, originados pelos sentimentos do doente, sejam recebidos e replicados pelo cérebro emocional do enfermeiro.

Carl Rogers, pai da teoria do cuidado centrado na pessoa, defende que para sermos enfermeiros empáticos é necessário que evidenciemos, permanentemente, sensibilidade para compreender as constantes mudanças que ocorrem na pessoa, a sua perceção do significado, sentimentos e emoções que vivencia. Segundo o autor, o profissional, ao ser empático, vive temporariamente no universo da pessoa, movendo-se delicadamente dentro dele sem julgar, perceber os significados que ele/ela quase não percebe, tudo isto sem tentar revelar sentimentos dos quais a pessoa não tem consciência, pois isto poderia ser muito ameaçador.

No processo de relação terapêutica entre enfermeiro, doente e sua família, torna-se fundamental que o enfermeiro compreenda e acolha a pessoa na sua singularidade, desprendendo-se de diagnósticos clínicos e julgamentos de valor com vista a identificar e satisfazer as necessidades da pessoa, promovendo o tratamento da mesma. 


\section{OBJETIVOS}

Neste estudo, formularam-se os seguintes objetivos de investigação:

$\mathrm{Ol}_{1}$ : Conhecer os níveis de empatia dos enfermeiros;

$\mathrm{Ol}_{2}$ : Conhecer os níveis dos traços de personalidade dos enfermeiros.

Colocaram-se ainda as seguintes hipóteses de investigação:

$\mathrm{HI}_{1}$ : Existem diferenças nos níveis de empatia de acordo com os dados socioprofissionais;

$\mathrm{H}_{2}$ : Existem diferenças nos níveis dos traços de personalidade de acordo com os dados socioprofissionais;

$\mathrm{HI}_{3}$ : Existem relações entre as dimensões da empatia e dos traços de personalidade.

Para ir ao encontro dos objetivos e verificar as hipóteses de investigação, desenvolveu-se um estudo exploratório, transversal, descritivo e correlacional.

\section{AMOSTRA}

A amostra é constituída por 232 enfermeiros, dos quais 186 (80.2\%) são do género feminino, a faixa etária mais representada é a de idade inferior a 29 anos ( $n=85 ; 36.6 \%$ ), existe um igual número de profissionais casados/união de facto e solteiros ( $n=109 ; 47.0 \%$ ) e sendo a antiguidade de serviço mais frequente a de menos ou igual a 5 anos ( $n=82 ; 35.5 \%)$, conforme apresentado na Tabela 1.

Tabela 1. Caraterização da amostra

\begin{tabular}{cccc}
\hline \hline & & $\mathrm{n}$ & $\%$ \\
\hline \multirow{2}{*}{ Género } & Masculino & 46 & 19.8 \\
& Feminino & 186 & 80.2 \\
& Total & 232 & 100.0 \\
\multirow{3}{*}{ Classe de idade } & $<=29$ & 85 & 36.6 \\
& $30-35$ & 70 & 30.2 \\
\multirow{2}{*}{ Estado civil } & $36+$ & 77 & 33.2 \\
& Casado(a)/União de facto & 109 & 47.0 \\
& Solteiro(a) & 109 & 47.0 \\
Antiguidade no Serviço & Divorciado(a) & 14 & 6.0 \\
& $<=5$ & 82 & 35.5 \\
& $6-12$ & 72 & 31.2 \\
& $13+$ & 77 & 33.3 \\
\hline \hline
\end{tabular}

\section{METODOLOGIA}

\section{Instrumento de Recolha de Dados}

Utilizou-se um questionário online, constituído por três partes: Questões socioprofissionais; 0 Jefferson Scale of Physician Empathy, um questionário de autorrelato de vinte itens, respondidos numa escala de tipo Likert de sete pontos, medindo as dimensões: Compaixão, Tomada de perspetiva do doente e Capacidade de colocarse no lugar do doente (Hojat et al., 2001); e o Big-Five Inventory (BFI-44), um questionário de autorrelato de 44 itens, respondidos numa escala de tipo Likert de cinco pontos, que avalia os cinco grandes traços de personalidade: Extroversão, Amabilidade, Conscienciosidade, Neuroticismo, e Abertura a Novas Experiências (John et al., 1991).

\section{Tratamento Estatístico e Análise de Dados}

Os dados foram processados e as estatísticas calculadas, recorrendo ao programa estatístico IBM SPSS, na sua versão 23 para macOS. Foram calculados as médias e os respetivos desvios padrão para as oito dimensões em estudo: compaixão, perspetiva, colocar-se no lugar dos outros, extroversão, amabilidade, conscienciosidade, 
neuroticismo, e abertura a novas experiências. Procedeu-se à análise da normalidade da distribuição dos dados, para os vários grupos em estudo, através da observação visual dos gráficos de dispersão e pelo teste de ShapiroWilk (Ghasemi \& Zahediasl, 2012; Thode, 2002), bem como ao cálculo da homogeneidade das variâncias para cada dimensão e respetivo grupo socioprofissional, para definir os testes correlacionais a utilizar, paramétricos ou não-paramétricos, de acordo com o recomendado em vários estudos recentes (Qualls et al., 2010; Van Hoek et al., 2019; Winkens et al., 2017). Nos casos em que existia diferenças estatisticamente significativas, foi também calculado o tamanho de efeito através do $d$ de Cohen (Cohen, 1988; Sullivan \& Feinn, 2012). Para averiguar a existência de relações entre as oito dimensões em estudo, procedeu-se ao cálculo do coeficiente de correlação rho de Spearman, seguindo também as recomendações mais recentes (Bishara \& Hittner, 2012).

\section{Questões Éticas}

Foram cumpridos todos os procedimentos éticos elencados na Declaração de Helsínquia (World Medical Association, 2013), tendo sido garantida a participação anónima e voluntária e explicado aos participantes 0 objetivo do estudo. A participação só era possível após aceitação explícita.

\section{RESULTADOS}

Para responder ao primeiro objetivo: conhecer os níveis de empatia dos enfermeiros, calcularam-se as pontuações médias e respetivos desvios padrão, conforme o género (Tabela 2). Como pode ser observado, os respondentes do género masculino pontuam mais alto na dimensão perspetiva, sendo as respondentes do género feminino as que respondem mais alto nas outras duas dimensões da empatia, a compaixão e o colocar-se no lugar do doente.

Tabela 2. Valores médios e desvios padrão para as dimensões da empatia

\begin{tabular}{cccc}
\hline \hline Género & Compaixão & Perspetiva & Colocar-se \\
\hline Masculino $(\mathrm{n}=46)$ & $5.78 \pm 0.858$ & $5.54 \pm 1,199$ & $5.33 \pm 0,874$ \\
Feminino $(\mathrm{n}=186)$ & $6.05 \pm 0,903$ & $5.45 \pm 1.551$ & $5.60 \pm 0,968$ \\
Total $(\mathrm{n}=232)$ & $6.00 \pm 0.899$ & $5.47 \pm 1.486$ & $5.55 \pm 0.955$ \\
\hline
\end{tabular}

Para responder ao segundo objetivo: conhecer os níveis dos traços de personalidade dos enfermeiros, calcularam-se as médias e respetivos desvios padrão para os cinco grandes traços de personalidade, sendo os resultados apresentados na Tabela 3, neste caso, divididos por género.

Tabela 3. Valores médios e desvios padrão para as dimensões dos traços de personalidade

\begin{tabular}{lccccc}
\hline Género & Extroversão & Amabilidade & $\begin{array}{c}\text { Consciencio- } \\
\text { sidade }\end{array}$ & $\begin{array}{c}\text { Neuroticis- } \\
\text { mo }\end{array}$ & Abertura \\
\hline $\begin{array}{l}\text { Masculino } \\
(\mathrm{n}=46)\end{array}$ & $3.73 \pm 0.501$ & $3.85 \pm 0.447$ & $4.00 \pm 0.498$ & $2.35 \pm 0.587$ & $3.61 \pm 0.530$ \\
$\begin{array}{l}\text { Feminino } \\
(\mathrm{n}=186)\end{array}$ & $3.51 \pm 0.631$ & $3.98 \pm 0.514$ & $3.94 \pm 0.539$ & $2.45 \pm 0.481$ & $3.39 \pm 0.635$ \\
$\begin{array}{l}\text { Total } \\
(\mathrm{n}=232)\end{array}$ & $3.55 \pm 0.613$ & $3.95 \pm 0.504$ & $3.95 \pm 0.504$ & $3.95 \pm 0.531$ & $3.43 \pm 0.620$ \\
\hline \hline
\end{tabular}

Quanto às primeiras duas hipóteses de investigação: existem diferenças nos níveis de empatia de acordo com os dados socioprofissionais; e, existem diferenças nos níveis dos traços de personalidade de acordo com os dados socioprofissionais, foram encontradas somente diferenças estatisticamente significativas para a variável 
independente género e as dimensões compaixão e extroversão, conforme apresentado na Tabela 4. Em ambos os casos o tamanho de efeito (Cohen, 1988) é pequeno $(0.20 £ d<0.50)$.

Tabela 4. Resultados Análise Inferencial

\begin{tabular}{lccccc}
\hline \hline Género & Extroversão & Amabilidade & $\begin{array}{c}\text { Consciencio- } \\
\text { sidade }\end{array}$ & $\begin{array}{c}\text { Neuroticis- } \\
\text { mo }\end{array}$ & Abertura \\
\hline $\begin{array}{l}\text { Masculino } \\
(\mathrm{n}=46)\end{array}$ & $3.73 \pm 0.501$ & $3.85 \pm 0.447$ & $4.00 \pm 0.498$ & $2.35 \pm 0.587$ & $3.61 \pm 0.530$ \\
$\begin{array}{l}\text { Feminino } \\
(\mathrm{n}=186)\end{array}$ & $3.51 \pm 0.631$ & $3.98 \pm 0.514$ & $3.94 \pm 0.539$ & $2.45 \pm 0.481$ & $3.39 \pm 0.635$ \\
$\begin{array}{l}\text { Total } \\
(\mathrm{n}=232)\end{array}$ & $3.55 \pm 0.613$ & $3.95 \pm 0.504$ & $3.95 \pm 0.504$ & $3.95 \pm 0.531$ & $3.43 \pm 0.620$ \\
\hline
\end{tabular}

Finalmente, para verificar a terceira hipótese: existem relações entre as dimensões da empatia e dos traços de personalidade, procedeu-se ao cálculo dos coeficientes de correlação rho de Spearman (Tabela 5). Verifica-se que existem correlações positivas, estatisticamente significativas e moderadas $(0.39<r<0.70)$ entre várias das dimensões, e uma correlação negativa e fraca $(0.09<r<0.40)$ entre a dimensão neuroticismo e várias das outras dimensões em estudo. Contudo, em doze das 27 situações, as correlações não são estatisticamente significativas, provando-se, desta forma, somente parcialmente a terceira hipótese de investigação.

Tabela 5. Coeficientes de correlação de Spearman

\begin{tabular}{|c|c|c|c|c|c|c|c|}
\hline & Perspetiva & $\begin{array}{c}\text { Colocar- } \\
\text { se }\end{array}$ & $\begin{array}{l}\text { Extrovers } \\
\text { ão }\end{array}$ & $\begin{array}{c}\text { Amabilid } \\
\text { de }\end{array}$ & $\begin{array}{l}\text { Consciens } \\
\text { io-sidade }\end{array}$ & $\begin{array}{l}\text { Neuroti- } \\
\text { cismo }\end{array}$ & Abertura \\
\hline$\overline{\text { Compaixão }}$ & 0.036 & $0.481^{* *}$ & -0.042 & 0.044 & -0.029 & -0.124 & -0.051 \\
\hline Perspetiva & 1.000 & -0.054 & $0.458^{* *}$ & $0.596^{* *}$ & $0.562^{* *}$ & -0.128 & $0.563^{* *}$ \\
\hline Colocar & & 1.000 & -0.025 & -0.009 & -0.100 & $-0.161^{*}$ & -0.073 \\
\hline Extroversão & & & 1.000 & $0.422^{* *}$ & $0.481^{* *}$ & $-0.292^{* *}$ & $0.522^{* *}$ \\
\hline Amabilidade & & & & 1.000 & $0.572^{* *}$ & $-0.299^{* *}$ & $0.487^{* *}$ \\
\hline $\begin{array}{l}\text { Conscienciosidad } \\
\text { e }\end{array}$ & & & & & 1.000 & $-0.237^{* *}$ & $0.503^{* *}$ \\
\hline Neuroticismo & & & & & & 1.000 & $-.166^{*}$ \\
\hline
\end{tabular}

A correlação é significativa no nível 0,01 (bilateral).**

A correlação é significativa no nível 0,05 (bilateral).*

\section{DISCUSSÃO}

Comparando os resultados com o estudo de Aguiar, Salgueira, Frada e Costa (2009), os respondentes do nosso estudo pontuam mais baixo na dimensão perspetiva ( $\mathrm{M}_{\text {Aguiar }}=5.77$; $\left.\mathrm{DP}_{\text {Aguiar }}=.70\right)$ e mais alto na dimensão colocar ( $M_{\text {Aguiar }}=5.06$; DP Aguiar=.99). Na dimensão compaixão as pontuações são quase idênticas.

No que concerne os cinco traços de personalidade, não se encontram diferenças consideráveis com outros estudos recentes (Martins et al., 2017; Rey \& Extremera, 2014).

\section{CONCLUSÕES}

As várias evidências consultadas são unânimes no que concerne à importância de atitudes empáticas na prestação dos cuidados de saúde, em concreto na profissão de enfermagem. Constatamos que apesar de existirem modelos teóricos para compreender 0 desenvolvimento da empatia, são escassas as práticas que visem a aprendizagem e treino, sobretudo nos cursos de licenciatura. 
0 modelo de Watson é adotado em diversas instituições de saúde, hospitais, centros médicos e universidades com cursos na área da saúde, nos EUA e no México. 0 Watson Caring Science Institute foi criado pela própria autora para divulgação dos princípios da teoria e auxílio na implantação e manutenção dos pressupostos nos serviços de saúde, além de disponibilizar programas de treino dirigidos a profissionais, promover pesquisas e eventos científicos.

É unânime a ideia de que que a empatia proporciona tanto a satisfação dos doentes quanto a dos enfermeiros, todavia a sua articulação teórico-prática na enfermagem e nas demais áreas da saúde ainda é escassa. Baseados nesta premissa a inclusão destes conteúdos na formação inicial de profissionais de saúde apresenta-se como uma prioridade, sendo que já dispomos de formação pós graduada.

0 foco dos cuidados de saúde ainda se encontra ancorado no modelo biomédico e nos avanços tecnológicos. Apesar de muito se falar e refletir sobre a relação de ajuda enfermeiro doente, existe um hiato na interligação à prática. E continua a existir uma desvalorização na prática clínica da dimensão humanística na prestação dos cuidados. Se bem que em eventos científicos são apresentadas evidências que comprovam a magnitude de práticas de Humanitude, compaixão e empatia na melhor recuperação dos doentes, devendo fazer parte do plano de cuidados.

Muito se reflete sobre a falta de compaixão, a falta de comunicação terapêutica, a falta de apoio na experiência de sofrimento! Refletir sobre a Empatia inserida na Teoria de Jean Watson mostra-nos com clareza o quanto a Enfermagem precisa aprimorar a sua prática para preencher a lacuna do real cuidado, cumprir a sua missão e evoluir como ciência. A sustentação teórica e a valorização de aspetos humanísticos podem contribuir fortemente para uma maior magnitude da assistência dos cuidados de Enfermagem, e, consequentemente, para melhor recuperação dos doentes.

Estamos convictos e corroboramos com Watson, que, a presença efetiva da empatia é capaz de qualificar os processos assistenciais, que, por sua vez, necessitam estar ancorados em referenciais teóricos coerentes com os pressupostos humanísticos. Sendo desta forma possível construir um contexto de cuidado harmonioso que constantemente valorize o outro. Torna-se imperativo o desenvolvimento de projetos que promovam a aprendizagem e 0 treino destas práticas.

\section{REFERÊNCIAS BIBLIOGRÁFICAS}

Aguiar, P., Salgueira, A., Frada, T., \& Costa, M. J. (2009). Empatia Médica: tradução, validação e aplicação de um instrumento de medição. X Congresso Internacional Galego-Português de Psicopedagogia, 3705-3716.

Bishara, A. J., \& Hittner, J. B. (2012). Testing the significance of a correlation with nonnormal data: Comparison of Pearson, Spearman, transformation, and resampling approaches. Psychological Methods, 17(3), 399417. https://doi.org/10.1037/a0028087

Chalifour, J. (2008). A intervenção terapêutica: os fundamentos existencial-humanistas da relação de ajuda. Lusodidacta.

Cohen, J. (1988). Statistical power analysis for the behavioral sciences. Erlbaum. https://doi.org/10.1111/14678721.ep10768783

Ghasemi, A., \& Zahediasl, S. (2012). Normality tests for statistical analysis: A guide for non-statisticians. International Journal of Endocrinology and Metabolism, 10(2), 486-489.

Goleman, D. (1995). Emotional intelligence. Bantam.

Hojat, M., Gonnella, J. S., Nasca, T. J., Mangione, S., Vergare, M., \& Magee, M. (2002). Physician empathy: Definition, components, measurement, and relationship to gender and specialty. American Journal of Psychiatry, 159(9), 1563-1569. https://doi.org/10.1176/appi.ajp.159.9.1563

Hojat, M., Mangione, S., Nasca, T. J., Cohen, M. J. M., Gonnella, J. S., Erdmann, J. B., Veloski, J., \& Magee, M. (2001). The Jefferson Scale of Physician Empathy: Development and preliminary psychometric data. Educational and Psychological Measurement, 61(2), 349-365. https://doi.org/10.1177/00131640121971158 
John, 0., Donahue, E., \& Kentle, R. (1991). The Big Five Inventory - Versions 4a and 54. Journal of Research in Personality, 37(6), 504-528. https://doi.org/10.1016/S0092-6566(03)00046-1

Martins, S., Galvão, A., \& Pinheiro, M. (2017). Social entrepreneurship, psychological coaching as a developer of competences. III Congresso Ibero-Americano de Empreendedorismo, Energia, Ambiente e Tecnologia Social, 413-418.

Mcdonald, N. M., \& Messinger, D. S. (2011). The Development of Empathy : How, When, and Why. Em A. Acerbi, J. A. Lombo, \& J. J. Sanguineti (Eds.), Moral Behavior and Free Will: A Neurobiological and Philosophical Aprroach (pp. 341-368). If Press.

Ordem dos Enfermeiros. (2011). Regulamento do Perfil de Competências do Enfermeiro de Cuidados Gerais. Ordem dos Enfermeiros.

Peplau, H. E. (1997). Peplau's theory of interpersonal relations. Nursing Science Quarterly, 10(4), 162-167. https://doi.org/10.1177/089431849701000407

Qualls, M., Pallin, D. J., \& Schuur, J. D. (2010). Parametric versus nonparametric statistical tests: The length of stay example. Academic Emergency Medicine, 17(10), 1113-1121. https://doi.org/10.1111/j.15532712.2010.00874.x

Rey, L., \& Extremera, N. (2014). Positive psychological characteristics and interpersonal forgiveness: Identifying the unique contribution of emotional intelligence abilities, Big Five traits, gratitude and optimism. Personality and Individual Differences, 68(May), 199-204. https://doi.org/10.1016/j.paid.2014.04.030

Rogers, C. R. (1975). Empathic: An Unappreciated Way of Being. The Counseling Psychologist, 5(2), 2-10. https://doi.org/10.1177/001100007500500202

Sullivan, G. M., \& Feinn, R. (2012). Using Effect Size - or Why the P Value Is Not Enough. Journal of Graduate Medical Education, 4(3), 279-282. https://doi.org/10.4300/JGME-D-12-00156.1

Thode, H. C. (2002). Testing for Normality. Marcel Dekker, Inc.

Van Hoek, G., Portzky, M., \& Franck, E. (2019). The influence of socio-demographic factors, resilience and stress reducing activities on academic outcomes of undergraduate nursing students: A cross-sectional research study. Nurse Education Today, 72(July 2018), 90-96. https://doi.org/10.1016/j.nedt.2018.10.013

Watson, J. (1979). Nursing: The philosophy and science of caring. Nursing administration quarterly, 3(4), 8687.

Winkens, B., Engelen, G. J. J. A., Verhey, F. R. J., Schols, J. M. G. A., Collet, J., \& de Vugt, M. E. (2017). Wellbeing of nursing staff on specialized units for older patients with combined care needs. Journal of Psychiatric and Mental Health Nursing, 25(2), 108-118. https://doi.org/10.1111/jpm.12445

World Medical Association. (2013). World Medical Association Declaration of Helsinki Ethical Principles for Medical Research Involving Human Subjects (Vol. 310, Número 20, pp. 2191-2194). World Medical Association. https://doi.org/10.3917/jib.151.0124 